\title{
Erratum
}

Manuelle Medizin 2019·57:122-123 https://doi.org/10.1007/s00337-019-0513-y Online publiziert: 13. März 2019

(c) Springer Medizin Verlag GmbH, ein Teil von Springer Nature 2019

\section{Tab. 1 Interrater-Reliabilität}

Reliabilität

Berechnung Intraklassenkorrelationskoeffi-

zient

Differenz fLSR

0,500

Differenz aKrot

0,843

Auf.BI

0,821

RKonv. re

0,554

RKonv. li

0,514

Berechnung Kappa

Inkl.Rot

0,242

mTA

0,592

aKrot aktive Kopfrotation in Rückenlage, $f L S R$ modifizierte frontale Labyrinthstellreaktion, Auf.Bl. Kopfaufrichtung in Bauchlage, RKonv reaktive Rumpfkonvexität bei Kopfrotation, re rechts, li links, Inkl.Rot passive Kopfrotation in Inklination, ITA muskuläre Tonusasymmetrie

Die Onlineversion des Originalartikels ist unter https://doi.org/10.1007/s00337-019-0491-0 zu finden.
Robby Sacher ${ }^{1} \cdot$ Dana Loudovici-Krug ${ }^{2} \cdot$ Marc Wuttke' $^{1}$ Martin Knüdeler ${ }^{1}$

${ }^{1}$ Praxis für Manuelle Medizin, Dortmund, Deutschland

${ }^{2}$ Institut für Physiotherapie, Forschungsberatungsstelle MM, Universitätsklinikum Jena, Jena, Deutschland

\section{Erratum zu: Ein 4-Item- Symmetriescore zur Identifikation und} Verlaufskontrolle von infantilen Haltungs- und Bewegungsasymmetrien - KiSS

\section{Erratum zu:}

Manuelle Medizin 2019

https://doi.org/10.1007/s00337-019-

0491-0

Sehr geehrte Leserin, sehr geehrter Leser,

die Autoren der Publikation „Ein 4-ItemSymmetriescore zur Identifikation und Verlaufskontrolle von infantilen Haltungs- und Bewegungsasymmetrien KiSS“ haben nach erneuter kritischer Bewertung der Reliabilitätswerte des Symmetriescores teilweise Unstimmigkeiten festgestellt.
Betroffen sind die Intraklassenkorrelationskoeffizienten (ICC) der Differenzen der beiden ersten Scoreitems, d.h. die frontale Labyrinthstellreaktion (fLSR) sowie die aktive Kopfrotation (aKRot).

Die bereits publizierten Tab. 1, 2 und 3 zeigen anstatt des ICC für Interrater-, Intrarater- und Test-Retest-Reliabilitäten Cronbachs $a$. Dies hat jedoch keine negativen Auswirkungen auf die Gültigkeit und Relevanz des Symmetriescores.

Die hier aufgeführten $\bullet$ Tab. 1, 2 und 3 geben die vollständigen Werte mit den korrigierten Items fLSR und aKRot an.

Tab. 2 Intrarater-Reliabilität

\begin{tabular}{|c|c|c|c|c|c|c|}
\hline & Rater 1 & Rater 2 & Rater 3 & Rater 4 & Rater 5 & Rater 6 \\
\hline \multicolumn{7}{|c|}{ Berechnung Intraklassenkorrelationskoeffizient } \\
\hline Differenz fLSR & 0,892 & 0,754 & 0,902 & 0,696 & 0,783 & 0,977 \\
\hline Differenz aKrot & 0,889 & 0,893 & 0,920 & 0,814 & 0,940 & 0,917 \\
\hline Auf.Bl & 0,955 & 0,942 & 0,776 & 0,924 & 0,988 & 0,997 \\
\hline RKonv. re & 0,844 & 0,942 & 0,861 & 0,878 & 0,832 & 0,956 \\
\hline RKonv. li & 0,747 & 0,790 & 0,743 & 0,941 & 0,786 & 0,922 \\
\hline \multicolumn{7}{|c|}{ Berechnung Kappa } \\
\hline Inkl.Rot & 0,647 & 0,895 & 0,442 & 1,000 & c & 1,000 \\
\hline mTA & 1,000 & 1,000 & 0,778 & 1,000 & 0,743 & 1,000 \\
\hline
\end{tabular}

c constant, nicht berechenbar, „Division durch Null“ durch konstanten Wert, aKrot aktive Kopfrotation in Rückenlage, $f L S R$ modifizierte frontale Labyrinthstellreaktion, Auf.BI. Kopfaufrichtung in Bauchlage, RKonv reaktive Rumpfkonvexität bei Kopfrotation, re rechts, li links, Inkl. Rot passive Kopfrotation in Inklination, $m T A$ muskuläre Tonusasymmetrie 


\section{Tab. 3 Test-Retest-Reliabilität}

\begin{tabular}{|c|c|c|c|c|c|c|}
\hline & Rater 1 & Rater 2 & Rater 3 & Rater 4 & Rater 5 & Rater 6 \\
\hline \multicolumn{7}{|c|}{ Berechnung Intraklassenkorrelationskoeffizient } \\
\hline Differenz fLSR & 0,656 & 0,612 & 0,519 & 0,524 & 0,471 & 0,502 \\
\hline Differenz aKrot & 0,633 & 0,365 & 0,479 & 0,418 & 0,582 & 0,246 \\
\hline Auf.Bl & 0,803 & 0,802 & 0,785 & 0,787 & 0,765 & 0,605 \\
\hline RKonv. re & 0,364 & 0,308 & 0,225 & 0,148 & 0,405 & 0,202 \\
\hline RKonv. li & 0,308 & 0,504 & 0,607 & 0,674 & 0,327 & 0,152 \\
\hline \multicolumn{7}{|c|}{ Berechnung Kappa } \\
\hline Inkl.Rot & 0,600 & 0,500 & 0,263 & c & c & 1,000 \\
\hline mTA & 0,909 & 0,500 & 0,600 & 0,664 & 0,408 & 0,583 \\
\hline
\end{tabular}

Wir bitten um Beachtung der korrigierten Tabellen und entschuldigen uns für den Fehler.

Die Autoren

\section{Korrespondenzadresse}

\section{Robby Sacher}

Praxis für Manuelle Medizin

Freistuhl 3, 44137 Dortmund, Deutschland post@manmed.info
Möchten Sie einen Beitrag für Manuelle Medizin einreichen?

Wir freuen uns, dass Sie unsere Zeitschrift Manuelle Medizin mitgestalten möchten.

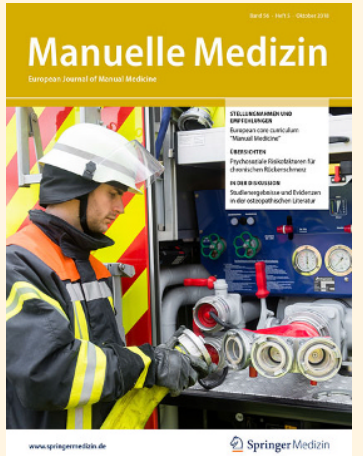

Für folgende Rubriken können Manuskripte eingereicht werden:

- Übersichten

- Originalien

- Kasuistiken

Um Ihnen bei der Manuskripterstellung behilflich zu sein, haben wir für unsere Autoren ausführliche Autorenleitfäden und Musterbeiträge für die verschiedenen Rubriken zusammengestellt.

Diese und weitere Hinweise zur Manuskripterstellung finden Sie online unter dem Menüpunkt „Hinweise für Autoren“ unter www.ManuelleMedizin.springer.de

Bitte reichen sie Ihre Manuskripte über den Editorial Manager ein. Wählen Sie hierzu auf der Zeitschriftenhomepage www.ManuelleMedizin.Springer.de den Navigationspunkt "Für Autoren" und anschließend "Manuskript online einreichen".

Bei Fragen zur Einreichung wenden Sie sich bitte an unser Editorial Office:

MaMe_office@springernature.com

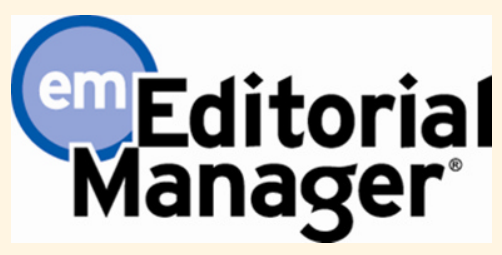

Wir freuen uns auf Ihre Beiträge! Ihre Redaktion von Manuelle Medizin 